\title{
Mesh morphing and response surface analysis: Quantifying sensitivity of vertebral mechanical behavior
}

\author{
Sigal I. A., and Whyne C. M. \\ Version Post-Print/Accepted Manuscript \\ Citation Sigal IA, Whyne CM. Mesh morphing and response surface analysis: \\ (published version) quantifying sensitivity of vertebral mechanical behavior. Ann Biomed \\ Eng. 2010 Jan;38(1):41-56. doi: 10.1007/s10439-009-9821-z. PMID: \\ 19859809
}

Publisher's Statement The final published version of this article is available at Springer via https://dx.doi.org/10.1007/s10439-009-9821-z.

\section{How to cite TSpace items}

Always cite the published version, so the author(s) will receive recognition through services that track citation counts, e.g. Scopus. If you need to cite the page number of the TSpace version (original manuscript or accepted manuscript) because you cannot access the published version, then cite the TSpace version in addition to the published version using the permanent URI (handle) found on the record page. 


\title{
Mesh morphing and response surface analysis: Quantifying sensitivity of vertebral mechanical behaviour
}

\author{
Sigal Ian A. A,*, Whyne Cari M ${ }^{\mathrm{A}, \mathrm{B}}$ \\ A Orthopaedic Biomechanics Laboratory, Sunnybrook Health Sciences Centre and \\ B Institute for Biomaterials and Biomedical Engineering, University of Toronto, \\ Toronto, Ontario, Canada.
}

\author{
Short Title: Vertebral mesh morphing and response surface analysis. \\ * Correspondence: Ian A. Sigal \\ Orthopaedic Biomechanics Laboratory, Sunnybrook Health Sciences Centre \\ 2075 Bayview Avenue, UB19, \\ Toronto, Ontario M4N 3M5, Canada \\ email: isigal@gmail.com \\ phone/fax: (503) 4135308 \\ Supported by the Canadian Institutes of Health Research \\ Note - Ian A Sigal is now a Research Associate at: \\ Ocular Biomechanics Laboratory, \\ Devers Eye Institute, \\ Portland, Oregon. USA.
}

Key Words: Morphing, response surface methods, parameterization, sensitivity analysis, vertebra, biomechanics, finite element modeling

Proprietary Interest: None

Number of Words: 5992

For submission to Annals of Biomedical Engineering 


\section{Abstract}

Vertebrae provide essential biomechanical stability to the skeleton. In this work novel morphing techniques were used to parameterize three aspects of the geometry of a specimen-specific finite element (FE) model of a rat caudal vertebra (process size, neck size, and end-plate offset). Material properties and loading were also parameterized using standard techniques. These parameterizations were then integrated within an RSM framework and used to produce a family of FE models. The mechanical behavior of each model was characterized by predictions of stress and strain. A metamodel was fit to each of the responses to yield the relative influences of the factors and their interactions. The direction of loading, offset and neck size had the largest influences on the levels of vertebral stress and strain. Material type was influential on the strains, but not the stress. Process size was substantially less influential. A strong interaction was identified between dorsal-ventral offset and dorsal-ventral off-axis loading. The demonstrated approach has several advantages for spinal biomechanical analysis by enabling the examination of the sensitivity of a specimen to multiple variations in shape, and of the interactions between shape, material properties and loading. 


\section{Introduction}

Vertebrae are an essential component in the provision of structural stability to the skeleton, however these bones are highly affected by pathologic processes such as osteoporosis and metastatic involvement. Vertebrae are the most common location for osteoporotic fractures and represent the location of the highest incidence of metastatic disease in the skeleton 13,36,55. The implications of the loss of vertebral structure and the resultant compromise of stability represent significant factors in terms of maintaining mobility and quality of life for patients with vertebral pathologies. Thus, in order to successfully predict and prevent fracture it is important to identify the relative impact of factors which affect vertebral response. Methods used to investigate stress and strain patterns in healthy and pathologic vertebrae, and assess the strength of the vertebral body have included numerical modeling, in vitro (cadaveric) mechanical testing and the use of preclinical in vivo models $18,24,26,30,42,48,49$.

Predicting the impact of pathologies on individual bones is difficult, in part because of the range of geometries, material distributions and applied loadings, but also because the effects of these factors are likely not independent. Finite element (FE) models allow representation of complex systems that occur in vivo and which are difficult to study with analytical or experimental techniques. Particularly useful is the construction of FE models such that aspects of their geometry, mechanical properties and loading can be varied by specifying a few high-level parameters. Using parametric FE methods it is possible to produce families of models designed to isolate and study specific effects independently.

FE models are often subdivided into generic models (developed to represent the general geometry and material properties of a population) and specimen-specific models (tailored to represent details of an individual specimen). Generic models can be difficult to initially develop but can be designed to facilitate parameterization. However, generic models cannot, by definition, provide information about the response of a particular specimen. Specimen-specific models can do this, but understanding the effects of individual parameters with such models remains a challenge, especially with respect to geometry. Therefore,

traditionally, parametric analysis has been carried out using either generic models, which allow variations of geometry, materials and loading, but which provide relatively vague predictions for a particular specimen, or using sets of specimen-specific models, each of which, allows variations in loading, and limited variations in materials, but whose predictions apply more directly to the specimen. The power of predictions made with specimen-specific models increases with the number of specimens, however, producing multiple specimenspecific models can be expensive, time consuming and difficult. Despite recent advances in automation, producing multiple robust models remains one of the main challenges for FE sensitivity studies 21, 39, 41, 45, 53.

The number of configurations that have to be analyzed to determine factor influences increases rapidly with the number of factors. This can be a challenge even for fully parameterized generic models, and it worsens for specimen-specific models which often require substantial computational resources for solution 
and analysis. The traditional one-factor-at-a-time (OFAT) method of evaluating factor influences is often used because it is relatively simple to implement and clear to analyze ${ }^{16,17}$, however, it is inefficient in the amount of information obtained from each model, and does not allow the evaluation of potential interactions between factors 4, 16,32. A response surface method (RSM) is a more efficient method to determine factor influences, independently and in interaction, as has been demonstrated in its application in many fields 4, 6, 8, 17, $27,29,31,37,52,54$. A further advantage of an RSM approach is that it produces metamodels, which are analytic phenomenological descriptions of the response as a function of the factors.

We propose that it is possible to combine the benefits of generic and specimen-specific modeling through the use of morphing techniques to alter the geometry of a FE model in a controlled and systematic way suitable for parametric study. This study aims to apply novel morphing techniques to parameterize the geometry of a specimen-specific model of a rat vertebra and to use these parameterizations in a sensitivity analysis within an RSM framework to derive metamodels and determine the relative influence of geometric, material property and loading factors on the mechanical response of the vertebra. 


\section{Methods}

\section{$\underline{2.1 \text { General strategy }}$}

The design of the study (Figure 1) consisted of generating a baseline model of a rat caudal vertebra, parameterizing the model geometry (using morphing techniques), material properties and applied loads (Table 1), with which a family of FE models with various combinations of the parameterized factors was produced according to a response surface design. To each of the responses we then fit an empirical metamodel, from which we determined the relative influences of the factors and their interactions on the vertebral mechanical behaviour. Pre and post-processing were done using Amira 3.1.1 (Mercury computer systems, USA); FE analysis using Abaqus 6.4 (Dassault systems, France); and RSM design and analysis using Design-Expert 7 (Stat-Ease, USA).

\subsection{The baseline model}

Model geometry was reconstructed from $\mu \mathrm{CT}$ images scanned at $17.5 \times 17.5 \times 17.5 \mu \mathrm{m} /$ voxel resolution (GE Explore Locus, General Electric Company, Fairfield, USA). Imaging was done with the X-ray source at $90 \mu \mathrm{A}$ and $80 \mathrm{kV}$, with 907 views covering $360^{\circ}$ of rotation. Reconstruction of the volume from the X-ray projection was done with the GE Explore Locus Recon utility to $17.5 \mu \mathrm{m} /$ voxel. The images were processed following a standard semi-automated segmentation (thresholding, filling and smoothing). The model volume was meshed with 4-node tetrahedral elements and the interior mesh iteratively smoothed and relaxed using Laplace's algorithm until the changes in nodal locations were smaller than $1 / 10 \mu \mathrm{m}$. (Figure 2)

\subsection{Parameterization}

2.3.1 Geometric Morphing Two distinct morphing techniques were utilized to parameterize three aspects of the baseline geometry. Offset (the vertebral rostral-caudal curvature) and neck size (the cross-sectional area at the thinnest vertebral section) were morphed using deformation vectors defined through analytical functions. Process size (the volume of the vertebral processes) was morphed using deformation vectors defined with a landmark-based method. The deformation vectors were then parameterized and used to translate the nodes of the baseline model, producing a corresponding model with the desired variations in geometry. The techniques were implemented using a combination of custom and standard Amira modules.

2.3.1.1 Offset: The $X$ and $Z$ Cartesian axes were aligned with the ventral-dorsal and the rostralcaudal axes of the vertebral model, respectively, and the origin $(0,0,0)$ with the vertebral centroid. The deformation function (in $\mu \mathrm{m}$, where the model coordinates are also in $\mu \mathrm{m}$ ): 


$$
f_{\text {offset }}(x, y, z)=\left(\frac{\mu_{1}}{1+e^{\frac{-z}{\mu_{2}}}}, 0,0\right) \quad\left(\mu_{1}=600 \mu \mathrm{m}, \mu_{2}=1000 \mu \mathrm{m}\right)
$$

was then used to define a relative displacement, or offset, of the rostral end. $\mu_{1}$ and $\mu_{2}$ control the magnitude and the width of the offset, respectively. The parameters were chosen to provide a smooth deformation, generally contained in the central one half of the vertebral height (i.e. leaving the shape of the caudal and rostral ends unchanged). The deformation is a function of $\mathrm{Z}$ only, but deforms only on $\mathrm{X}$. Note that the original geometry is asymmetric in offset, whereby the caudal and rostral ends are not aligned. Hence changes in the ventral direction increase the natural offset (to $20 \%$ ventral offset), whereas changes in the dorsal direction first decrease the offset, then increase it (to 10\% dorsal, Figure 1).

2.3.1.2 Neck size: We altered the cross-sectional area of the vertebra at its smallest point (the neck) by defining the following deformation function (in $\mu \mathrm{m}$, where the model coordinates are also in $\mu \mathrm{m}$ ):

$$
f_{\text {necksize }}(x, y, z)=\left(\mu_{3} e^{-\mathrm{x} \mu_{4}\left(\frac{z}{\mu_{5}}\right)^{2}}, \mu_{3} e^{-\mathrm{y} \mu_{4}\left(\frac{z}{\mu_{5}}\right)^{2}}, 0\right) \quad\left(\mu_{3}=35 \mu \mathrm{m}, \mu_{4}=2, \text { and } \mu_{5}=3000 \mu \mathrm{m}\right)
$$

where $\mu_{3,} \mu_{4}$, and $\mu_{5}$ are parameters that control the magnitude and distribution of the deformation. The deformation was a function of all $\mathrm{X}, \mathrm{Y}$ and $\mathrm{Z}$, but does not deform in Z (Figure 1). The parameters were chosen to vary the cross-sectional area from $45 \%$ to $177 \%$ of the value in the baseline model.

2.3.1.3 Process Size: The processes of the rat vertebra were shrunk/enlarged using deformation vectors defined through a Bookstein thin-plate spline method ${ }^{7}$. This method requires the definition of corresponding landmarks for the initial (before deforming) and final (after deforming) shapes (Figure 2). The initial landmarks were placed manually on the baseline geometry ( 3 on the larger processes, 2 on the smaller ones). The final landmarks were defined using cross sections, and the vectors normal to the model surface. From both sets of landmarks the thin plate spline method produces a warp field over the whole space. To constrain the deformation to the process regions an auxiliary 300 landmarks were defined randomly over the surface of the model, excluding the processes. The location of the auxiliary landmarks was unchanged between source and target. This also effectively eliminated cross-deformation between process, simplifying setting deformation scale for each process. To produce the exact desired changes in volume of each process (from $50 \%$ to $150 \%$ of the volume in the baseline model), the transformations obtained from the Bookstein method were parameterized linearly. The Bookstein transformations of all the process were combined to produce a deformation field controllable by a single parameter. 
2.3.1.4 Combining the geometric deformations: Once the deformation fields for each of the factors have been defined, they were combined into a single transformation by adding the deformations linearly:

$$
f_{\text {total }}=\alpha \cdot f_{\text {offset }}+\beta \cdot f_{\text {necksize }}+\gamma \cdot f_{\text {processsiz }}
$$

where each of the parameters $\alpha, \beta$ and $\gamma$ control the magnitude (and sense) of the deformations related to one factor. A linear combination of the deformations works best when the deformations are independent from one another. A simple way to ensure this is to base all the deformations on the geometry of the baseline specimen. If the deformations are not independent, the scaling constant may turn to scaling functions, which are difficult to calibrate and may not permit addition of the deformations linearly, complicating and reducing the generality of the analysis.

2.3.2 Loading direction and boundary conditions In the baseline model a purely axial compressive distributed load of $25 \mathrm{~N}$ was applied to the nodes on the rostral vertebral end plate. Nodal loads were weighted by element areas so as to apply a homogeneous force on the surface with normal and tangential components. In altering the load application, the force vectors on each node were decomposed into their Cartesian components and scaled to keep the total force constant while varying the angle of the force applied. The load was varied $20^{\circ}$ in each of the dorsal-ventral and lateral directions, producing two independent parameters. The nodes on the exterior surface of the caudal end-plate were constrained to zero rostral-caudal (Z) displacements. The node nearest the centroid of this surface was constrained in all directions to provide a reference frame for the solutions and prevent translations and rotations.

2.3.3 Material Properties Two types of material properties were analyzed (Figure 1): intensity-based and homogenized. In the intensity-based definition each element was assigned material properties based on $\mu \mathrm{CT}$ voxel intensity using the following conversion equation 22, 50, 51:

$$
E_{t}[M P a]=20.173 \times\left(6.83 \times 10^{-4} \times I \times+9.713 \times 10^{-1}\right)^{2.46}
$$

Where $\mathrm{I}$ is the element's representative intensity, and $\mathrm{E}_{\mathrm{t}}$ the Young's modulus in the transverse direction in MPa. This lead to elements being assigned moduli between 12.5 and $3662 \mathrm{MPa}$. All elements were assigned a Poisson ratio of $0.3^{22}$.In the homogenized definition, elements were assigned to one of three materials (or bins): cortical bone, trabecular bone, and very soft incompressible tissue (to represent marrow and the regions of the growth plate which have low intensity in the CT scan). The main difference between the two types of material analyzed was the number of bins: 500 for the intensity-based type, three for homogenized type. The moduli in the homogenous definition were assigned such that the overall response (total response force) of the baseline model was the same as in the intensity-based definition. This allowed the effects of inhomogeneity to be assessed independently of overall model stiffness. Anisotropy varied linearly from perfectly isotropic for the elements with highest modulus, representing cortical bone, to transversely isotropic 
for the elements with the lowest modulus in which the modulus in the axial $(Z)$ direction was $3 x$ higher than in the transverse $(\mathrm{X}, \mathrm{Y})$ plane ${ }^{15}$. Note that the material property definitions are generated from the baseline specimen.

\subsection{Simulation and mesh quality}

The nodes of the baseline mesh were translated according to the total deformation prescribed by the combination of the three geometry deformations described above. The interior of the deformed mesh was then iteratively smoothed and relaxed again using Laplace's algorithm until the changes in nodal locations were smaller than $1 / 10 \mu \mathrm{m}$. The elements were initially 4-noded (for mesh generation), but for solution they were converted to 10-noded tetrahedra by adding midside nodes.

We judged the quality of the mesh of all the models generated through evaluation of the number of distorted elements (ABAQUS). Element size, aspect ratio and the angle between faces were used to judge element quality 1,14, 46. A mesh refinement study was conducted on the baseline model. Once sufficient element resolution was determined, the resolution was increased to allow for the higher resolution requirements of other configurations. This yielded a baseline specimen-specific mesh consisting of 52,323 10node tetrahedral elements and 77,010 nodes (230,430 degrees of freedom) (Figures 1 and 2). After simulation and analysis, cases with particularly high levels of stress or strain were refined and solved again to verify that the default resolution was sufficient. In every case it was. Mesh resolution was increased by splitting each tetrahedra into 8 smaller tetrahedral. To separate mesh resolution from material property assignments and focus on the numerical accuracy, the smaller tetrahedra inherited the material properties of the parent mesh.

\section{$\underline{2.5 \text { Responses }}$}

The mechanical response of each vertebral model to loading was characterized through the Von Mises stress, and maximum and minimum principal (tensile and compressive) strains. The population distribution of each of these responses was calculated and characterized through the median and peak (50 $0^{\text {th }}$ and $95^{\text {th }}$ percentiles). The analysis was restricted to a region of interest comprising the central $66 \%$ of the vertebra, to reduce artefacts which may arise due to the superior and inferior growth plates, as unlike in humans, rat vertebral growth plates remain open throughout their life cycle ${ }^{22}$.

2.5.1 Transforming the responses The response variables were transformed, as is standard in response surface methods 4,32 , which allows the factor effects to be added in an unbiased fashion, and improves the distribution of the residuals (see below the section on metamodel fitting). We used the traditional Box-Cox analysis and plot method to determine the optimal transformation for each response ${ }^{4,32}$. For all the responses, the optimal transformation was a base 10 logarithm. This is equivalent to expressing the response 
in decibels, and is a common transformation for strain and stress in bioengineering ${ }^{17,44}$. For plotting, the responses were converted back to the original scale.

\subsection{RSM strategy}

2.6.1 Experimental design An inscribed spherical rotatable central composite design on five continuous factors (geometry and loading) was used to choose the parameter combinations for this study 4,32 . The design was then duplicated to add the material type as a categorical factor with two levels, for a total of 92 runs. Eight additional runs were inserted randomly into the sequence to verify that there was no pure error (such as drift), as it should be in deterministic analyses.

2.6.2 Metamodel fitting A mathematical relationship representing a response surface, or metamodel, was regressed for each of the responses. This surface was a second order polynomial of the form:

$$
g\left(x_{1}, x_{2}, \ldots, x_{6}\right)=\beta_{0}+\sum_{i=1}^{6} \beta_{i} x_{i}+\sum_{i=1}^{6} \sum_{j=1}^{i} \beta_{i j} x_{i} x_{j}+\varepsilon
$$

where $g$ is the function fit to a response, $\beta^{\text {'s }}$ are the regression coefficients to be estimated, $x$ 's the factors, and $\varepsilon$ the residual. As the equation above is nonlinear in the x's (factor levels), but linear in the $\beta$ 's the polynomial response surface is a type of linear regression model. The number of runs in the experiment design was more than the minimum needed to fit the second order polynomial, and in this sense the response function was overdefined. The data remaining after fitting the model was used to obtain measures of quality of the fit, i.e. how well the curve represents the data. Quality of fit is most commonly calculated using the coefficient of determination $\left(\mathrm{R}^{2}\right)$, however this coefficient is susceptible to artifacts (e.g. its value increases with the number of data points or with the range of the data). Thus the adjusted and predicted $\mathrm{R}^{2}$ were used which are less sensitive to such artifacts 4,32 . We also computed the signal-to-noise ratio as the ratio of the range of the predicted values to the average prediction error ${ }^{4}$.

2.6.3 Identifying factor influences The relative influence of the factors was determined by comparing the factor and interaction contributions to the sum of squares corrected by the mean, as is traditionally computed in an analysis of variance (ANOVA). We followed the tradition in RSM of coding all factors to the range -1 to +1 , which allows for a fair comparison independent of factor scales, units or range size $4,8,32$. Factors were included in the model if their effects were statistically significant (as deemed by ANOVA with $\mathrm{p}<0.001$ ), or by hierarchy ${ }^{3,34}$. We evaluated using larger $\mathrm{p}$ values (results not shown). This increased the number interactions in the model that were statistically significant but which contributed little, thereby only increasing complexity. The influential factors, and interactions are illustrated using contour-level and interaction plots. We also generated perturbation plots to aid in the interpretation of the effects of factor variations to the baseline. Perturbation plots are akin to one-factor-at-a-time analysis and are therefore 
relatively easy to interpret. But these plots have to be interpreted carefully when interactions are present, since variations in the reference point could lead to substantially different curves. 


\section{Results}

\subsection{Morphing and parameterization}

The morphing algorithms were incorporated successfully into a pipeline to parameterize offset, neck size, process size, material properties and two variations in loading. This pipeline allowed efficient preparation of models with the desired combinations of factors. In all models the number of distorted elements was very small (between 0.02 and $0.2 \%$ of the elements). This proportion is on the same order as what has been observed with model morphing using other algorithms $5,25,28,43,46$. This means that the deformations were sufficiently smooth and did not compromise usability for FE modeling and analysis. Pure error was at least six orders of magnitude smaller than the responses and therefore considered zero.

\subsection{Sensitivity analysis}

The results of the RSM analysis (quality of fit and sums of squares corrected by the mean) for all the responses and all the factors parameterized generated excellent fits with adjusted $\mathrm{R}^{2}$ values between 0.9670 and 0.9954 , predicted $\mathrm{R}^{2}$ values between 0.9475 and 0.9956 , residuals below $2 \%$ of the mean and high signal to noise ratios (Table 2). Taken together these results reinforce the idea that the fits captured the responses adequately. The sum of squares for model (third line from the top in Table 2) were several times larger than the rest of the contributions, consistent with the fact that the response ranges over the factor space studied were relatively small compared with the response for the baseline model. To compare the relative influence of the factors, the fraction (in percent) of the contribution to the total sum of squares was computed 44,56 (Table 3). The coefficients of the metamodels (the polynomial functions fit to each) of the responses are listed in the Appendix.

Neck size was the most influential factor on both median and peak stress (because the largest stresses occur at the smallest cross section, or neck of the vertebral body) (Table 3). Recall the models were fit to transformed responses, and therefore even the linear components of the models do not imply a linear effect of the factor on the response. Particularly strong were the contributions of the quadratic components of the neck-size and both directions of loading. In the perturbation plots for median von Mises stress (Figure 3) neck size demonstrated a complex nonlinear effect.

Variations in the loading led to changes in the stress (Figure 4). As would be expected from beam bending theory, off-axis loading led to bending of the vertebra and increased the stresses in all sides, but more in the side opposite to the loading. Under off-axis loading the strains in one side become slightly more tensile, and in the other slightly more compressive. The von Mises stress aggregates both forms of strain, while discounting hydrostatic forces, into a composite, always positive, quantity. The consequence is that the lowest von Mises stresses occur under axial loading. In the perturbation plots for median von Mises stress (Figure 3) we notice the quadratic nature of the dependence of stress on the loading direction. Asymmetry is 
evident in the response in the lack of overlap between the lines. In the response surface, Peak stress had a minimum near the center of the loading direction factor space with contour levels of stress forming ellipses centered on this minimum, meaning that the baseline configuration is a point of minimum peak stress. The ellipses were slightly tilted, which demonstrates the asymmetry in the response to loading. The response surface for the median stresses had a saddle shape, meaning that the baseline configuration is a point of minimum median stress in one direction, and of maximum stress in another. However, for both median and peak stresses the slope of the response surface is small near the center, suggesting that small variations in the factors may also translate to small effects on the response.

The contribution of offset was found to be relatively small (Table 3), however a statistically significant effect was found for the interaction between the offset and the dorsal-ventral loading (BD) (Figure 5). Independently, deviations in loading from the axial direction led to increases in stress, whereas conversely, deviations from the center, in offset, led to decreases in stress. When the vertebra is offset ventrally, tilting the loading ventrally as well reduces the peak stress. In contrast, when the vertebra is offset dorsally the same tilting of the loading increases the peak stress. Thus, the effect of offset on the level of stress depended strongly on the direction of loading as illustrated in the interaction plot (Figure 4). Switching loading from one direction to the other can reverse the effect of changes in offset. Similarly, the effects of variations in loading can be reversed depending on the offset.

The material model had a strong influence on the strains, especially on the median compressive strains (more than half the variance). This was, in part, because other factors had modest influence on the median compressive strain, increasing the fraction of variance contributed by the material model. Lower strains were produced when the material was set as intensity based than when it was homogenized (Figure 4). As expected from the axial compressive loading, higher magnitudes were seen in compressive as compared to tensile strains.

Process size was overall the least influential input factor (Figure 2 and Table 3). Its largest relative influence was on median von Mises stress and median tensile strain, but these were still less than $5 \%$ of the total variations observed. Increasing process size led to small increases in median stress. 


\section{Discussion}

This paper demonstrates a novel application of morphing techniques to effectively parameterize specimen specific bony geometry. This has several advantages for biomechanical analysis in enabling the examination of model sensitivities on a wider scale. The parameterizations gained through morphing substantially increase the reach of sensitivity studies by making it possible to study not only the role of factors representing geometry, but also of interactions between geometry, material properties and loading. Interactions between factors have been shown to be fundamental for understanding other biomechanical systems ${ }^{38,44}$. However, until now the analysis of interactions involving geometry was only possible through generic models or through statistical analysis of sets of specimens. Approximations necessary for producing generic models may omit aspects essential to its response and producing sets of specimen-specific models does not allow the parameters of interest to be isolated with each model presenting variations in several factors (some of which may not be readily apparent). Morphing avoids generic approximations and allows factor isolation at limited computational expense.

Parameterizations generated utilizing a baseline model yield altered geometries useful for studying the biomechanical consequences of variations of the specific baseline specimen, i.e. studying its factor neighborhood. These variations could be the consequence of aging, disease, injury, or of approximations made for experimentation or simulation. It is conceivable that specimens will differ in their sensitivity to the factors, and therefore a general analysis of sensitivity over a whole population may not adequately predict specimen sensitivity.

Multiple models produced through morphing the mesh are relatively easy to compare as they share common elements, nodes and connectivity and morphing facilitates maintenance of material properties and boundary conditions from one model to another. This is particularly useful for studies with complex boundary conditions, and further motivates their use in modeling of more complex spinal mechanics when contact surfaces (i.e. facets), and soft tissue attachments (i.e. discs and ligaments) are included and can save considerable time and effort in pre and post-processing.

Combining the parameterizations with RSM techniques allows the fitting of metamodels to the responses which facilitates the interpretation of the model results. Metamodels, as algebraic expressions are relatively easy to interpret. By using RSM techniques, it is possible to quantify and distinguish linear and quadratic components of the effects of the parameterized factors on the responses, which provides a more comprehensive perspective of the sensitivity. RSM techniques also substantially reduce the number of simulations required compared with traditional OFAT techniques ${ }^{4,32}$.

Metamodels fit to the responses can also be used as surrogates of the modeling for predicting the responses quantitatively. Despite the metamodels being long equations, numerically they are simple to use for calculations, and can even, in some instances, replace the need to carry out FE simulations. As algebraic 
expressions, metamodels are also convenient because they allow for the calculation of integrals and derivatives, and identification of extrema or inflection points at relatively low cost ${ }^{6}$. For these and other useful properties, response surfaces have seen widespread application in describing systems and optimization 4,6 .

A strong interaction was identified between dorsal-ventral loading and dorsal-ventral offset.

Although only one statistically significant influential interaction was identified, that between offset and dorsalventral loading, this is important. First, because it underscores the value of having combined morphing of geometric factors and RSM techniques. If either of these techniques had not been included, this interactions would not have been identified. Second, because it allows the potential effects of other factor interactions to be dropped from further consideration, thereby simplifying future study designs. The size of the vertebral processes had a relatively modest effect, even under off-axial loading, in enlarged processes, or in thin vertebrae with large offset. Low stress levels show that only a modest amount of loading is carried by these processes. We expected that other modes of loading, i.e. off-axis loading, would transfer some of the loading to the processes, and increase their influence. This would have been apparent as an interaction between process size and off-axis angles. However, the magnitude of this interaction was minimal, which means that the details of process geometry are not essential for predicting the biomechanical response of the vertebra. One of the challenges during specimen-specific model reconstruction is capturing the process geometry. If it is not critical, then a relatively simple and approximate reconstruction may suffice.

When interactions are present the curves may depend on the choice of reference point, and therefore perturbation plots need to be interpreted carefully. We decided to include the plots in this work for three reasons: 1) the baseline model is a special one, the true specimen-specific case, and as such small deviations around this case are particularly interesting (for example, approximations in loading during testing can be approximated by perturbations); 2) the main characteristics of the curves noted in this section did not vary substantially with the choice of reference point (data not shown), and therefore that presenting the perturbation plots was unlikely to be misleading; 3) interpretation of perturbation plots is simple, yet they illustrate the key effects.

A common concern when fitting metamodels in RSM techniques is the choice of analytic function. There is no a priori reason to expect that a second order polynomial shall provide an adequate representation of the system, however, in practice second order polynomials often work 6, 27, 29, 37, 52, 54. The regressed function found through an RSM fit is not a functional relationship, but rather an approximation within defined bounds. The analysis provides a means to evaluate quality of fit and determined that the choice of the function was adequate for characterizing the caudal vertebral biomechanics. For the level of precision that we are extracting from the approximated surfaces we find the fits excellent. Increasing the complexity of the function could have provided for a closer approximation, but as always it is important to balance fidelity with 
simplicity. In addition, the metamodels will inherit the limitations of the FE models, and there is little benefit in increasing the accuracy of the fit to responses that may already imply an approximation.

Although in this work we successfully morphed an FE mesh of a rat vertebrae, our intention is to continue developing the techniques to morph more complex structures and in more intricate ways. The transformations useful for morphing are constrained by the need to preserve mesh quality. An alternative, which we have begun exploring ${ }^{47}$, is to apply the morphing techniques to the model surfaces only, which are more robust to deformation, followed by remeshing of the model volume after deformation. This takes time, and is less convenient for the comparison between models because the direct pairing between model volume meshes is lost.

Morphing techniques are relatively common within the fields of animation and computer graphics. Some ideas from these fields have been ported to biomechanics $19,20,35$, but to the best of our knowledge not been used for parameterizing models for sensitivity analysis. In free-form deformation (FFD) techniques, based on the ideas by Sederberg and Parry ${ }^{40}$, the baseline geometry is embedded in a control volume, which is deformed using a set of landmarks as a guide, passing the deformation to the embedded objects. FFD methods are a good alternative for morphing for sensitivity studies, however, they share some limitations with the methods presented in this work, most notably that the deformations involve some arbitrariness, and that their application still requires a considerable amount of work to determine adequate control volumes and landmarks to guide the deformation ${ }^{20}$. Another interesting use of morphing in biomechanics has been to use FE to deform a template model to a target ${ }^{9-11}$. In this technique nonlinear continuum mechanics are used, which guarantees a continuous mapping between source and target. The technique, however, has the problem that it requires a manual selection of material properties, which is arbitrary and has a strong effect on the deformation field 9,11 . Also, such mechanics-based methods have more demanding computational requirements than the methods presented here.

The methods have some limitations that deserve consideration. First is that there is some degree of arbitrariness in the deformations used to parameterize the geometry, although our choices were informed by an understanding of the anatomy and biomechanics of the vertebra 2, 12, 23, 46. Ideally morphometric data in which the nature and magnitude of the physiologic variations in shape would guide the deformations, however such information is not available for the rat caudal vertebra.

The models in this work were formed by tetrahedral elements (4-noded for pre and post-processing, and 10-noded for simulation). It would be preferable to use higher order elements in all steps, and avoid the approximations, as any inherent limitations of the original model will propagate through the sensitivity analyses. There is nothing, in theory, which prevents the application of the morphing techniques described here to other element types and for the proof-of-concept purposes of this work, we believe that linear tetrahedral elements suffice. 
The models were simulated using linear elastic material properties, which is a common approximation when simulating bones, as long as the load regime is not at a high rate, does not approach failure, and the scale of the analysis is large with respect to the trabecular beam sizes ${ }^{33}$. However, the material approximation is more limited in describing the behavior of the growth plates, which were not modeled independently. As such, the responses analyzed were restricted to the central region of the main vertebral body, away from the growth plates. The influence of material model was strong in the strains. This may be due to the way in which the homogenized material model was developed in which bins for moduli were chosen to produce a model that reacted with the same total force as the model with intensity-based materials, yielding equivalent stress. Strain had no such requirements and therefore it was affected more by the choice of material properties. Had we chosen the homogenized materials using a displacement (i.e. strain based) condition, then the material model may have influenced stress rather than strain. Both material types were based on the baseline model and material distributions were morphed by the same transformation defined by anatomical considerations. Once the baseline specimen-specific model was distorted, i.e. morphed to a new geometry, the material distribution is not necessarily applicable. It is conceivable that vertebra with different geometries may also have different distributions of materials, especially as bone adapts to physiologic loadings to which a specimen was subjected in vivo. As presented, therefore, the results on material type obtained in this study can only be used to conclude that it is important to use an intensity-based material type rather than the particular homogenized one we defined. Nevertheless, this work demonstrates that the morphing and RSM techniques can be used to determine the effects of material type, independently and in interaction. Interestingly, no interactions were identified between material model and other factors, suggesting that, for this particular system, it is possible to use the simplified homogenized materials and "correct" for this later. Again, there is nothing, in theory, which prevents using more complex material types. In future work it would be possible to vary the material properties independently of the geometry after morphing, but to do this requires a robust characterization of material distribution patterns.

In summary, the morphing and RSM analyses presented in this work have several advantages over conventional FE modeling. (1) The morphing methods allow parameterizing the geometry of specimenspecific FE models, allowing exploring variations that may arise during aging or disease, or through approximations in modeling. (2) Models related through morphing are easier to setup and analyze than unrelated models. (3) RSM is an efficient technique for exploring the factor space, and identifying the relative influences of factors, and their interactions. (4) Through RSM metamodels of the responses are produced that are relatively easy to analyze and may ultimately serve as surrogates for the FE models.

Applying the morphing and RSM techniques to the analysis of rat caudal vertebra mechanics, it was found that the direction of loading, offset and neck size had the largest influences on the levels of stress and strain within the vertebra. Material type was influential on the strains, but not the stress. Process size was substantially less influential. A strong interaction was identified between dorsal-ventral offset and dorsal- 
ventral off-axis loading. Understanding the relative importance of the factors and their interactions can be used to direct and simplify future investigations utilizing more complex spinal geometries (i.e. motion segments) and loading conditions.

The originality of this work lies in the application of morphing techniques to parameterize the geometry of a specimen-specific FE model useful in the field of orthopaedic biomechanics. We have demonstrated some of the substantial benefits to biomechanical analysis available when morphing-based parameterizations are implemented within an RSM technique. Morphing is common in animation, but not in biomechanics. Similarly, although RSM techniques are not new, they are also not standard, and it is our hope that their integration with morphing encourages their further use in orthopaedic biomechanics research.

\section{Acknowledgements}

Funding for this work was provided by the Canadian Institutes of Health Research (CIHR).

\section{References}

1. Abaqus. Abaqus v6.5.1 Online Reference Manual. Providence, RI, USA: Dassault Systems, 2006.

2. Akens MK, Hardisty MR, Wilson BC, Schwock J, Whyne CM, Burch S, and Yee AJ. Defining the therapeutic window of vertebral photodynamic therapy in a murine pre-clinical model of breast cancer metastasis using the photosensitizer BPD-MA (Verteporfin). Breast Cancer Res Treat 2009.

3. Anderson MJ, and Whitcomb PJ. DOE Simplified: Practical tools for effective experimentation. Productivity Press, 2000, p. 256.

4. Anderson MJ, and Whitcomb PJ. RSM Simplified: optimizing processes using response surface methods for design of experiments. Productivity Press, 2005, p. 292.

5. Bade R, Haase J, and Preim B. Comparison of fundamental mesh smoothing, algorithms for medical surface models. In: Visualization in Medicine, Theory, Algorithms, and Applications, edited by Preim B, and Bartz DMorgan Kaufmann, 2007.

6. Bhadra S, and Ganguli R. Aeroelastic optimization of a helicopter rotor using orthogonal array-based metamodels. AIAA journal 44: 1941-1951, 2006.

7. Bookstein FL. Describing a craniofacial anomaly: finite elements and the biometrics of landmark locations. Am J Phys Anthropol 74: 495-509, 1987.

8. Box GEP, Hunter JS, and Hunter WG. Statistics for experimenters: design, innovation, and discovery. Wiley-Interscience, 2005.

9. Brock KK, Dawson LA, Sharpe MB, Moseley DJ, and Jaffray DA. Feasibility of a novel deformable image registration technique to facilitate classification, targeting, and monitoring of tumor and normal tissue. Int J Radiat Oncol Biol Phys 64: 1245-1254, 2006.

10. Brock KK, Nichol AM, Menard C, Moseley JL, Warde PR, Catton CN, and Jaffray DA. Accuracy and sensitivity of finite element model-based deformable registration of the prostate. Med Phys 35: 4019-4025, 2008.

11. Brock KK, Sharpe MB, Dawson LA, Kim SM, and Jaffray DA. Accuracy of finite element model-based multi-organ deformable image registration. Med Phys 32: 1647-1659, 2005.

12. Burch S, Bisland SK, Wilson BC, Whyne C, and Yee AJ. Multimodality imaging for vertebral metastases in a rat osteolytic model. Clin Orthop Relat Res 454: 230-236, 2007. 
13. Cauley JA, Hochberg MC, Lui LY, Palermo L, Ensrud KE, Hillier TA, Nevitt MC, and Cummings SR. Long-term risk of incident vertebral fractures. Jama 298: 2761-2767, 2007.

14. Couteau B, Payan Y, and Lavallee S. The mesh-matching algorithm: an automatic 3D mesh generator for finite element structures. Journal of Biomechanics 33: 1005-1009, 2000.

15. Cowin SC, and Doty SB. Tissue Mechanics. New York: Springer, 2007.

16. Czitrom V. One-Factor-at-a-Time versus designed experiments. The american statistician 53: 126-131, 1999.

17. Dar FH, Meakin JR, and Aspden RM. Statistical methods in finite element analysis. J Biomech 35: 1155$1161,2002$.

18. Dimar JR, 2nd, Voor MJ, Zhang YM, and Glassman SD. A human cadaver model for determination of pathologic fracture threshold resulting from tumorous destruction of the vertebral body. Spine 23: 1209-1214, 1998.

19. Fernandez JW, Ho A, Walt S, Anderson IA, and Hunter PJ. A cerebral palsy assessment tool using anatomically based geometries and free-form deformation. Biomech Model Mechanobiol 4: 39-56, 2005.

20. Fernandez JW, Mithraratne P, Thrupp SF, Tawhai MH, and Hunter PJ. Anatomically based geometric modelling of the musculo-skeletal system and other organs. Biomech Model Mechanobiol 2: 139$155,2004$.

21. Gibson AP, Riley J, Schweiger M, Hebden JC, Arridge SR, and Delpy DT. A method for generating patient-specific finite element meshes for head $\backslash$ modelling. Phys Med Biol 48: 481-495, 2003.

22. Hardisty MR. Strain measurement in vertebral bodies by image registration. In: Institute of biomaterials and biomedical engineering. Toronto, Canada: University of Toronto, 2006.

23. Hardisty MR, and Whyne CM. Whole bone strain quantification by image registration: a validation study. J Biomech Eng 131: 064502, 2009.

24. Horst M, and Brinckmann P. 1980 Volvo award in biomechanics. Measurement of the distribution of axial stress on the end-plate of the vertebral body. Spine 6: 217-232, 1981.

25. Krause R, and Sander O. Automatic construction of boundary parametrizations for geometric multigrid solvers. Comput Vis Sci 9: 11-22, 2006.

26. Lavaste F, Skalli W, Robin S, Roy-Camille R, and Mazel C. Three-dimensional geometrical and mechanical modelling of the lumbar spine. J Biomech 25: 1153-1164, 1992.

27. Lee C-C, Lee C-C, Ku H-T, Chang S-M, and Chiang K-N. Solder joints layout design and reliability enhancements of wafer level packaging using response surface methodology. Microelectronics reliability 47: 196-204, 2007.

28. Lee CF, Chen PR, Lee WJ, Chen JH, and Liu TC. Three-dimensional reconstruction and modeling of middle ear biomechanics by high-resolution computed tomography and finite element analysis. Laryngoscope 116: 711-716, 2006.

29. Lendrem D, Owen M, and Godbert S. DOE (Design of experiments) in developmental chemistry: potential obstacles. Organic process research \& development 5: 324-327, 2001.

30. McBroom RJ, Hayes WC, Edwards WT, Goldberg RP, and White AA, 3rd. Prediction of vertebral body compressive fracture using quantitative computed tomography. J Bone Joint Surg Am 67: 12061214, 1985.

31. Meakin JR, Shrive NG, Frank CB, and Hart DA. Finite element analysis of the meniscus: the influence of geometry and material properties on its behaviour. Knee 10: 33-41, 2003.

32. Montgomery DC. Design and Analysis of Experiments. Wiley, 2004.

33. Mow VC, and Hayes WC. Basic Orthopaedic Biomechanics. Lippincott Williams \& Wilkins Publishers, 1997.

34. Nelder JA. The selection of terms in response-surface models -- How strong is the weak-heredity principle? The american statistician 52: 315, 1998.

35. O'Reilly MA, and Whyne CM. Comparison of computed tomography based parametric and patientspecific finite element models of the healthy and metastatic spine using a mesh-morphing algorithm. Spine (Phila Pa 1976) 33: 1876-1881, 2008.

36. Ortiz Gomez JA. The incidence of ertebral body metastases. Int Orthop 19: 309-311, 1995. 
37. Preetha B, and Viruthagiri T. Application of response surface methodology for the biosorption of copper using Rhizopus arrhizus. Journal of Hazardous Materials 143: 506-510, 2007.

38. Rekow ED, Harsono M, Janal M, Thompson VP, and Zhang G. Factorial analysis of variables influencing stress in all-ceramic crowns. Dent Mater 22: 125-132, 2006.

39. Roberts MD, and Hart RT. Shape adaptation of long bone structures using a contour based approach. Comput Methods Biomech Biomed Engin 8: 145-156, 2005.

40. Sederberg TW, and Parry SR. Free-Form deformation of solid geometric models. In: SIGGRAPH 86, edited by Evans DC, and Athay RJ. New York: ACM SIGGRAAPH, 1986, p. 151-160.

41. Shim VB, Pitto RP, Streicher RM, Hunter PJ, and Anderson IA. The use of sparse CT datasets for autogenerating accurate FE models of the femur and pelvis. J Biomech 40: 26-35, 2007.

42. Shirado O, Kaneda K, Tadano S, Ishikawa H, McAfee PC, and Warden KE. Influence of disc degeneration on mechanism of thoracolumbar burst fractures. Spine 17: 286-292, 1992.

43. Shontz SM, and Vavasis SA. A mesh warping algorithm based on weighted Laplacian smoothing. In: 12th International Meshing Roundtable. Santa Fe, NM: 2003, p. 147-158.

44. Sigal IA. Interactions between geometry and mechanical properties on the optic nerve head. Invest Ophthalmol Vis Sci 2009.

45. Sigal IA, Flanagan JG, Tertinegg I, and Ethier CR. Modeling individual-specific human optic nerve head biomechanics. Part I: IOP-induced deformations and influence of geometry. Biomech Model Mechanobiol 2008.

46. Sigal IA, Hardisty MR, and Whyne CM. Mesh-morphing algorithms for specimen-specific finite element modeling. J Biomech 41: 1381-1389, 2008.

47. Sigal IA, Yang H, Roberts MD, and Downs JC. Morphing methods to parameterize specimen-specific finite element model geometries. Journal of Biomechanics Submitted.

48. Silva MJ, Hipp JA, McGowan DP, Takenchi T, and Hayes WC. Strength reductions of thoracic vertebrae in the presence of transcortical osseous defects: Effects of defect location, pedicle disruption, and defect size. European Spine Journal 2: 118-125, 1993.

49. Suwito W, Keller TS, Basu PK, Weisberger AM, Strauss AM, and Spengler DM. Geometric and material property study of the human lumbar spine using the finite element method. J Spinal Disord 5: 5059, 1992.

50. Taddei F, Pancanti A, and Viceconti M. An improved method for the automatic mapping of computed tomography numbers onto finite element models. Med Eng Phys 26: 61-69, 2004.

51. Taddei F, Schileo E, Helgason B, Cristofolini L, and Viceconti M. The material mapping strategy influences the accuracy of CT-based finite $\backslash$ element models of bones: An evaluation against experimental measurements. Med Eng Phys 29: 973-979, 2007.

52. Vard JP, Kelly DJ, Blayney AW, and Prendergast PJ. The influence of ventilation tube design on the magnitude of stress imposed at the implant/tympanic membrane interface. Med Eng Phys 30: 154-163, 2008.

53. Viceconti M, and Taddei F. Automatic generation of finite element meshes from computed tomography data. Crit Rev Biomed Eng 31: 27-72, 2003.

54. Wang JL, Shirazi-Adl A, and Parnianpour M. Search for critical loading condition of the spine--a meta analysis of a nonlinear viscoelastic finite element model. Comput Methods Biomech Biomed Engin 8: 323-330, 2005.

55. Wong DA, Fornasier VL, and MacNab I. Spinal metastases: The obvious, the occult, and the impostors. Spine 15: 1-4, 1990.

56. Yao J, Salo AD, Lee J, and Lerner AL. Sensitivity of tibio-menisco-femoral joint contact behavior to variations in knee kinematics. J Biomech 41: 390-398, 2008. 


\section{Appendix}

The coefficients of the metamodels (Table A1) can be assembled into a function, such as

$\log _{10}($ Median von Mises stress $)=1.708+0.047 \times \mathrm{A}-0.003 \times \mathrm{B} \ldots-0.191 \times \mathrm{B} \times \mathrm{D}-0.045 \times \mathrm{B}^{2} \ldots+0.198 \times \mathrm{E}^{2}$. where $\mathrm{A}=$ Process size, $\mathrm{B}=$ Offset, etc. all coded from -1 to +1 . Predictions are in $\mathrm{MPa}$ for the stresses and in percentages for the strains. Note that the coding extends to the material model, although only the extreme values -1 and +1 are meaningful. Some of the elements included in the equations contribute little to the metamodel, and were included for completeness. If those elements are not included the equations become are simpler and predict essentially the same values. See Table 2 for measures of the quality of fit. To simplify comparison we report strain absolute values. 


\section{Figure captions}

Figure 1: General study strategy. A baseline specimen-specific FE model of a rat caudal vertebra was produced based on a $\mu \mathrm{C}$ T scans of a normal specimen. An experimental design was produced following a response surface method. The design prescribes the combinations of parameters to be studied. Morphing techniques were applied to parameterize three aspects of the baseline model geometry: process size (parameterized using a landmark-based technique), and offset and neck size (parameterized using analytic functions). Models were assigned materials, loading and boundary conditions and solved using the FE method. A region of interest was selected, and the response of the model characterized by predictions of stress and strain within that region. An empirical response surface, or metamodel, was fit to each of the responses using multivariate linear regression. Second order polynomials were chosen as the empirical models. The coefficients of the metamodels were then analyzed to determine the relative influence of the various factors parameterized, and their interactions.

Figure 2: Illustration of the landmark-based morphing technique used to vary process size. Dorsal (top row) and caudal (second rows) views of three semi-transparent models, with shrunk processes (left), the baseline (center), and with enlarged processes (right). The morphing was defined through landmarks manually placed on the surface of the vertebra processes (blue points). Using cross sectional views a corresponding set of landmarks was defined inside the processes (red points). A warping field was then computed that paired surface and interior landmarks (lines joining the blue and red points). The warping field was scaled linearly to produce the desired changes in process volume. Caudal views of the model exterior with elements outlined (third row). Cross sections at the level of the rostral processes colored according to the level of von Mises stress under purely axial load (bottom row). The morphing changes the size, but not the overall shape, of the processes without altering the rest of the vertebra. Note how the processes are under small loads, explaining why process shrinkage had only a minor effect on the level of stress.

Figure 3: Perturbation plots as a graphical representation of relative factor effects. Factors vary on the $\mathrm{X}$ axis from lowest to highest coded values. On the $\mathrm{Y}$ axis are different aspects of model response to loading: median von Mises stress and median tensile and compressive strain. This type of plot illustrates the effects of varying factors independently, i.e. one factor is varied, while other factors are kept constant at their baseline level. Horizontal lines indicate that a factor had little influence on the response, whereas a steep line indicates a strong influence. Since the responses were transformed (see text), even the responses for factors that have no interactions or quadratic components may not be straight lines. Median von Mises stresses were not sensitive to material type, and therefore only one plot is shown (with values the average of both material types). For strains, use of intensity-based materials reduced the levels of both modes of strain. Off-axis loading, independent of the direction, increased stress, with the lines not overlapping, evidencing asymmetry in vertebra shape (see Figure 4 for model cross sections under various loadings). Offset in any direction reduced stresses. Lateral and dorsal-ventral loadings had quadratic effects, and their lines do not overlap, evidencing an asymmetry in the vertebra shape. Overall process size and offset had the smallest effects, whereas loading had the largest. Most influential on the strains was the neck size, although interestingly its effects were different in tensile than in compressive strains. However, it is important to remember that the lines representing the effects of offset and dorsal-ventral loading can be misleading because the factors interact (Figure 5). To simplify comparison we report strain absolute values. Recall, however, that perturbation plots have to be interpreted carefully when interactions are present 
Figure 4: Effects of the material type and loading direction on the von Mises stress. Longitudinal (top) and transverse (bottom) cross sections of the baseline model colored according to the magnitude of von Mises stress. Two models with the baseline geometry and axial loading, with intensity-based (top left) and homogenized (top right) materials. Although the distributions are very similar, the model with homogenized materials presents slightly larger stresses, and somewhat larger stress gradients (see, for example, the edge of the deep blue central region, the edges are sharper on the homogenized case). Also noticeable is that the processes are under small loads in both models. Note the outlines of the growth and end plates. The effects of loading direction are illustrated with cross sections through the baseline geometry, at the level of the neck (bottom left), or through the caudal processes (bottom right). At the center is the case with axial loading, around are off-axis ( \pm 1$)$ loadings laterally and dorsal-ventral. Stresses were clearly minimal under perfectly axial loading. Under offaxis loadings the deformations are a combination of compression and bending. However, both of these modes contribute to the von Mises stress. Off-axis loading increased the load carried by the processes, but the stresses were still concentrated on the base of the process, and depended little on the process shape.

Figure 5: Effects of the interaction between offset and dorsal-ventral loading. There was a strong interaction between dorsal-ventral loading and offset. There are two traditional ways to illustrate the effects of an interaction like this, contour level plots (top) and so-called interaction plots (bottom). Median (left) and peak (right) stresses were affected differently by the interaction. Peak stress was minimum near the center of the factor space (the baseline). Contour levels of stress formed ellipses centered on this minimum. The ellipses were slightly tilted, explaining the asymmetry in effects of lateral and dorsal-ventral loading seen in the perturbation plots of Figure 3. The response surface for the median stresses had a saddle shape, with the inflection point at the center. This evidences a maximum in one direction, and a minimum in another. These directions were not perfectly aligned with the offset and dorsal-ventral loading. Interactions between factors are also often illustrated with interaction plots where non-parallel lines evidence an interaction and are relatively easy to distinguish. Cross lines, such as in these examples, are even clearer. For instance, it is easy to see that the effects of off-axis loading could be substantially different depending on the vertebra offset. Switching loading from one direction to the other leads to increases in stress for offset in one direction, and decreases for offset in the opposite direction. Similarly, the effects of variations in offset may depend strongly on the direction of loading. However, some characteristics of the interaction are more difficult to see in the interaction plots than on contour level plots. For example, from only the interaction plot lines it is difficult to form a mental image of the ellipses in the peak stresses, or the saddle shape on the median stresses. It is therefore common to evaluate both types of plot and pick the one that illustrates most clearly the important effects for the case at hand

Sigal et al., Vertebral mesh morphing and response surface analysis. 


\section{Table captions}

Table 1: Factors and their ranges. Five factors were treated as continuous, and one as categorical (materials). See text for details of the definition of each factor and a justification of the range. Offset is not symmetric because there is a natural offset in the vertebra as reconstructed.

Table 2: Sums of squares corrected by the mean and quality of fit for each of the responses. From the quality of fit measures we can see that the metamodels were excellent representations of the responses. All values, except those in italics, were statistically significant $(\mathrm{p}<0.001)$. Of the 15 possible two-factor interactions between the six factors, only the interaction between offset (B) and dorsal-ventral loading (D) was statistically significant. For analysis the responses were transformed by the base 10 logarithm. To simplify comparison we report all strain modes in absolute values.

Table 3: Graphical representation of the relative magnitude of the contributions to the sum of squares of Table 2. This table shows for each response (columns) the percentage that each factor (row) contributes to the sum of squares corrected by the mean in Table 2. A long bar means that the contribution of the factor to the response is large, and therefore that the factor has a strong effect. The contributions can be classified depending on whether they represent the independent effect of the factor (main factors), factor interactions (interaction), or quadratic contributions (curvature). Neck size $(\mathrm{C})$ and loading ( $\mathrm{E}$ and $\mathrm{F}$ ) had the strongest contributions. Process size (A), offset (B) and material model (F) had moderate contributions, although this varied across responses. 


\section{Tables}

\begin{tabular}{|c|c|c|c|c|}
\hline & \multirow{2}{*}{ Factor } & \multirow{2}{*}{$\begin{array}{l}\text { Coded } \\
\text { name }\end{array}$} & \multicolumn{2}{|c|}{ Factor range } \\
\hline & & & Low (-1) & High (+1) \\
\hline \multirow{3}{*}{ Geometry } & $\begin{array}{l}\text { Process size } \\
(\% \text { of initial volume })\end{array}$ & A & $150 \%$ & $50 \%$ \\
\hline & $\begin{array}{c}\text { Offset } \\
(\% \text { change in end-plate alignment } \\
\text { relative to the baseline })\end{array}$ & $\mathrm{B}$ & $\begin{array}{c}20 \% \\
\text { ventral }\end{array}$ & $\begin{array}{l}10 \% \\
\text { dorsal }\end{array}$ \\
\hline & $\begin{array}{c}\text { Neck size } \\
(\% \text { of initial neck area) }\end{array}$ & $\mathrm{C}$ & $45 \%$ & $177 \%$ \\
\hline \multirow{3}{*}{ Loading } & Dorsal-Ventral & $\mathrm{D}$ & $20^{\circ}$ ventral & $20^{\circ}$ dorsal \\
\hline & & & & \\
\hline & Lateral & $\mathrm{E}$ & $-20^{\circ}$ & $20^{\circ}$ \\
\hline
\end{tabular}

\begin{tabular}{|c|c|c|c|c|}
\hline & & & Level A & Level B \\
\hline $\begin{array}{c}\text { Material } \\
\text { properties }\end{array}$ & $\begin{array}{c}\text { Homogenized vs. } \\
\text { Intensity-based }\end{array}$ & F & $\begin{array}{c}\text { Intensity-based } \\
(500 \text { bins })\end{array}$ & $\begin{array}{c}\text { Homogenized } \\
\text { (3 bins) }\end{array}$ \\
\hline
\end{tabular}

Table 1: Factors and their ranges. Five factors were treated as continuous, and one as categorical (materials). See text for details of the definition of each factor and a justification of the range. Offset is not symmetric because there is a natural offset in the vertebra as reconstructed. 


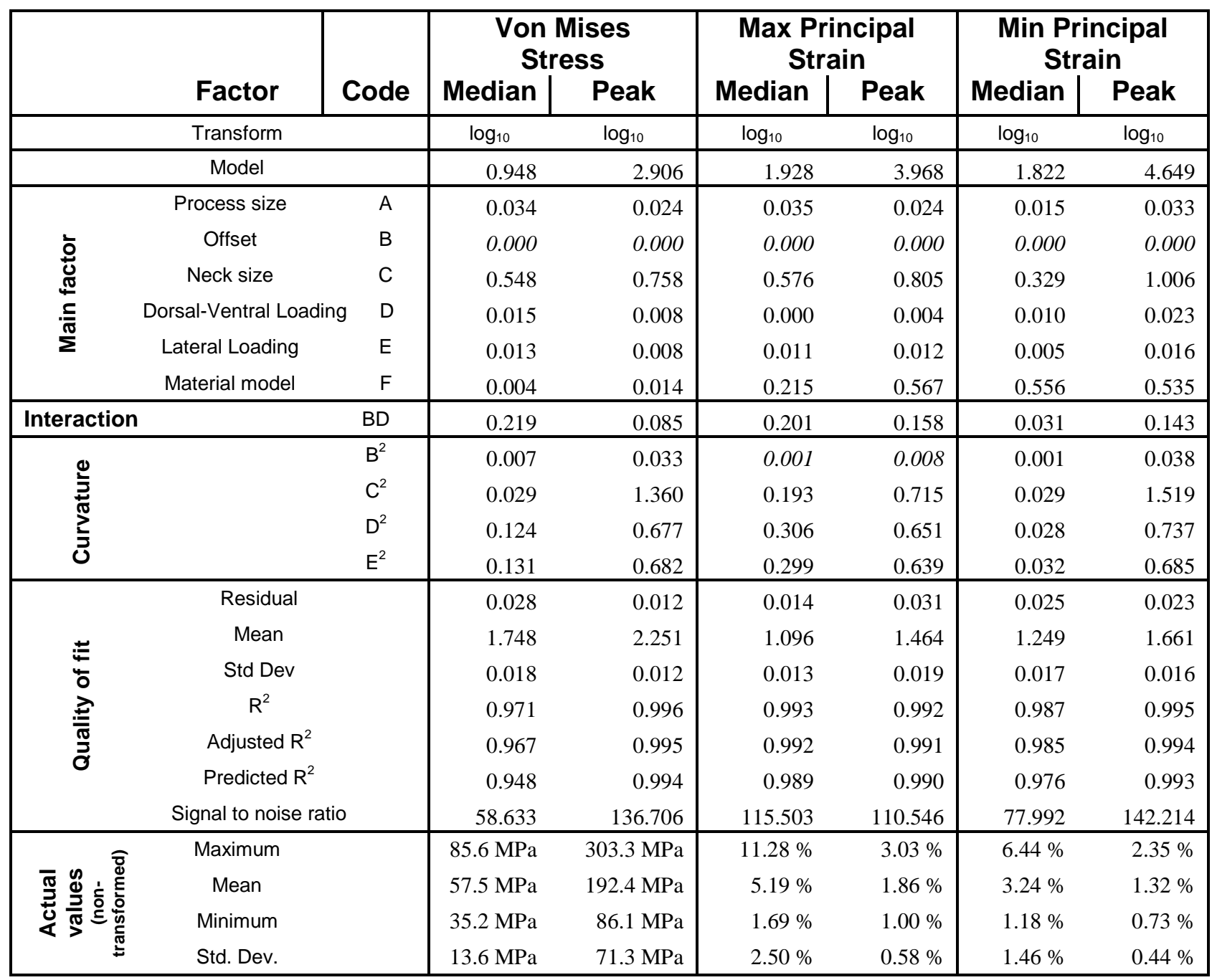

Table 2: Sums of squares corrected by the mean and quality of fit for each of the responses. From the quality of fit measures we can see that the metamodels were excellent representations of the responses. All values, except those in italics, were statistically significant $(\mathrm{p}<0.001)$. Of the 15 possible two-factor interactions between the six factors, only the interaction between offset (B) and dorsal-ventral loading (D) was statistically significant. For analysis the responses were transformed by the base 10 logarithm. To simplify comparison we report all strain modes in absolute values. 


\begin{tabular}{|c|c|c|c|c|c|c|c|c|}
\hline & \multirow[b]{2}{*}{ Factor } & \multirow[b]{2}{*}{ Code } & \multicolumn{2}{|c|}{ Von Mises Stress } & \multicolumn{2}{|c|}{ Max Prin Strain } & \multicolumn{2}{|c|}{ Min Prin Strain } \\
\hline & & & Median & Peak & Median & Peak & Median & Peak \\
\hline \multirow{6}{*}{ 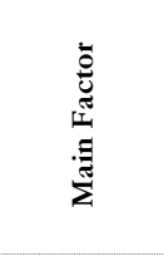 } & Process size & A & & & & & & \\
\hline & Offset & B & & & & & & \\
\hline & Neck size & c & & & & & & \\
\hline & Dorsal-Ventral Loading & $\mathrm{D}$ & & & & & & \\
\hline & Lateral Loading & E & & & & & & \\
\hline & Material model & $\mathrm{F}$ & & & & & & \\
\hline Interaction & & $B D$ & & & & & & \\
\hline$\cong$ & & $\mathrm{B}^{2}$ & & & & & & \\
\hline E & & $\mathrm{c}^{2}$ & & & & & & \\
\hline 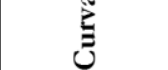 & & $\begin{array}{l}D^{2} \\
E^{2}\end{array}$ & & & & & & \\
\hline
\end{tabular}

Table 3: Graphical representation of the relative magnitude of the contributions to the sum of squares of Table 2. This table shows for each response (columns) the percentage that each factor (row) contributes to the sum of squares corrected by the mean in Table 2. A long bar means that the contribution of the factor to the response is large, and therefore that the factor has a strong effect. The contributions can be classified depending on whether they represent the independent effect of the factor (main factors), factor interactions (interaction), or quadratic contributions (curvature). Neck size (C) and loading (E and F) had the strongest contributions. Process size (A), offset (B) and material model (F) had moderate contributions, although this varied across responses. 


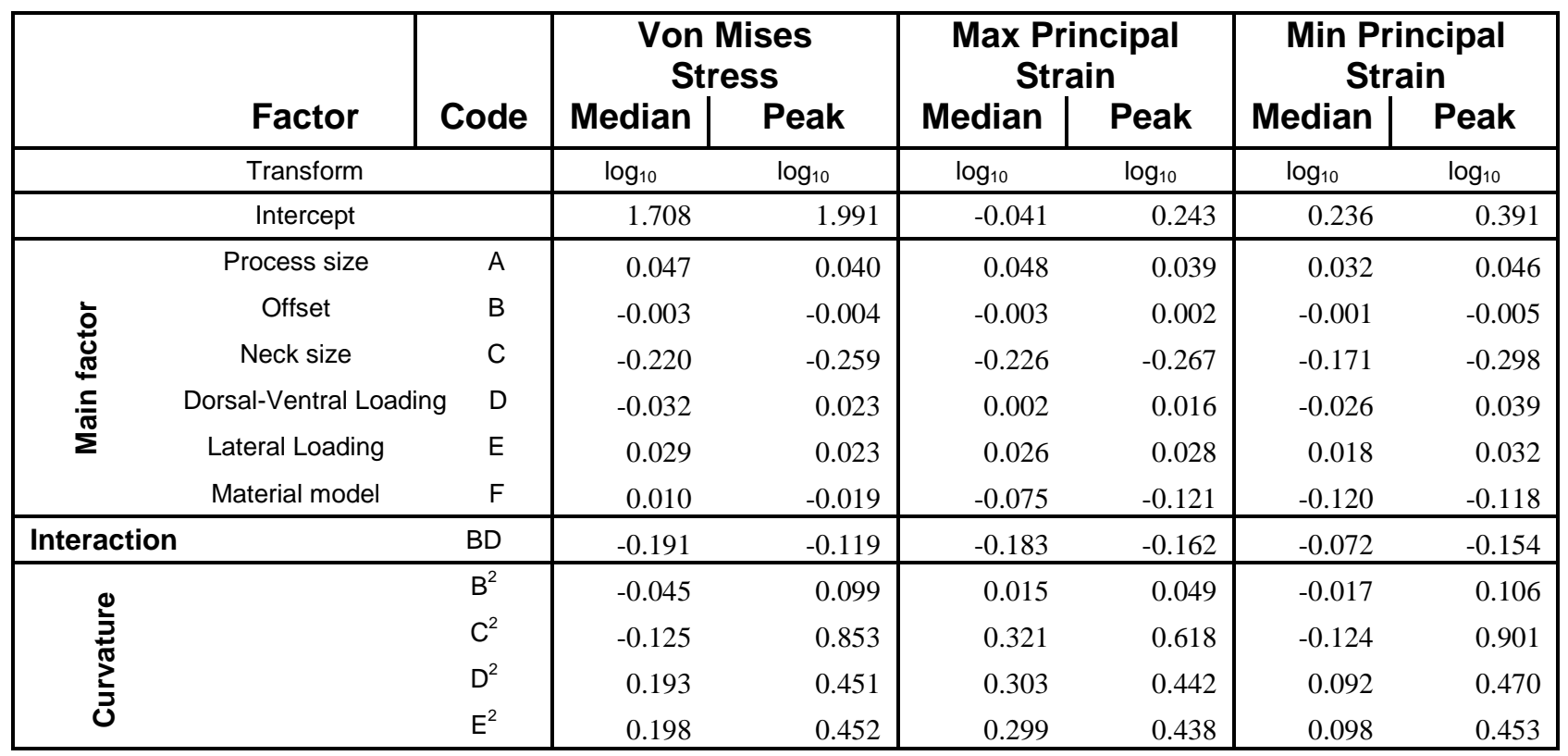

Table A1: Coefficients of the metamodel (the polynomial functions fit to each) of the responses. 


\section{Figures}

Figure 1: General study strategy. A baseline specimen-specific FE model of a rat caudal vertebra was produced based on a $\mu \mathrm{CT}$ scans of a normal specimen. An experimental design was produced following a response surface method. The design prescribes the combinations of parameters to be studied. Morphing techniques were applied to parameterize three aspects of the baseline model geometry: process size (parameterized using a landmark-based technique), and offset and neck size (parameterized using analytic functions). Models were assigned materials, loading and boundary conditions and solved using the FE method. A region of interest was selected, and the response of the model characterized by predictions of stress and strain within that region. An empirical response surface, or metamodel, was fit to each of the responses using multivariate linear regression. Second order polynomials were chosen as the empirical models. The coefficients of the metamodels were then analyzed to determine the relative influence of the various factors parameterized, and their interactions. 


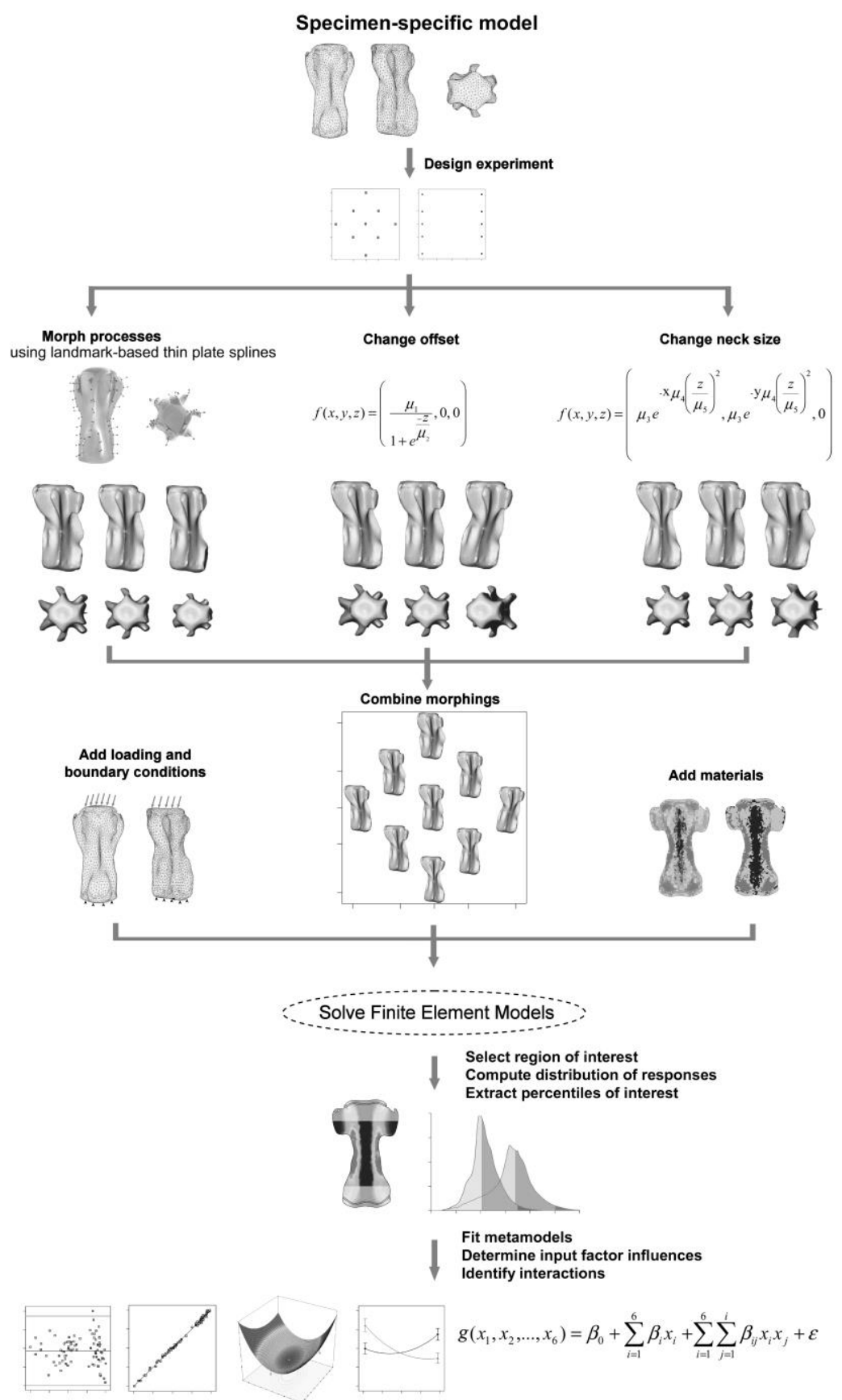

Sigal et al., Vertebral mesh morphing and response surface analysis. 

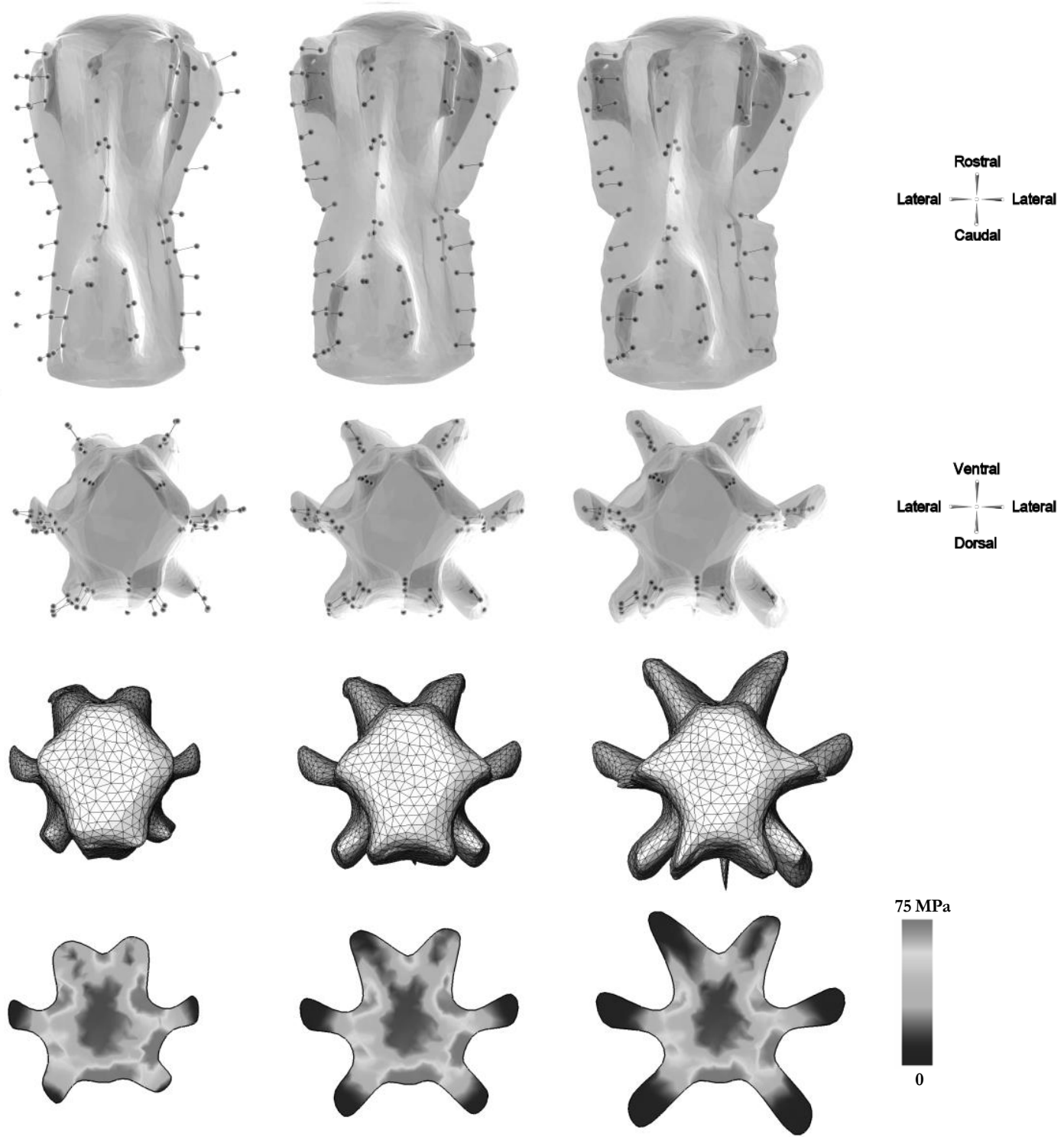

Figure 2: Illustration of the landmark-based morphing technique used to vary process size. Dorsal (top row) and caudal (second rows) views of three semi-transparent models, with shrunk processes (left), the baseline (center), and with enlarged processes (right). The morphing was defined through landmarks manually placed on the surface of the vertebra processes (blue points). Using cross sectional views a corresponding set of landmarks was defined inside the processes (red points). A warping field was then computed that paired surface and interior landmarks (lines joining the blue and red points). The warping field was scaled linearly to produce the desired changes in process volume. Caudal views of the model exterior with elements outlined (third row). Cross sections at the level of the rostral processes colored according to the level of von Mises stress under purely axial load (bottom row). The morphing changes the size, but not the overall shape, of the processes without altering the rest of the vertebra. Note how the processes are under small loads, explaining why process shrinkage had only a minor effect on the level of stress. 


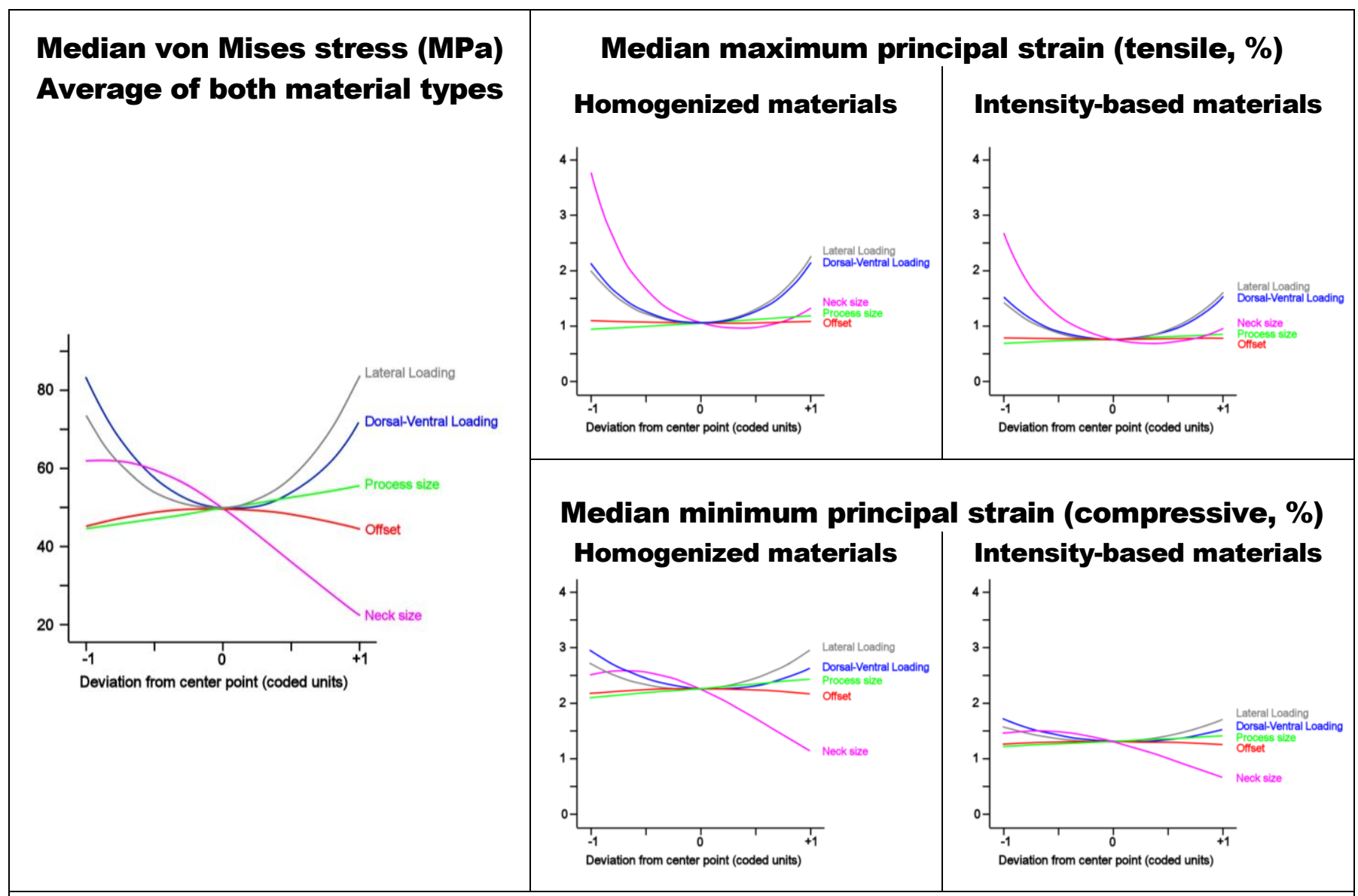

Figure 3: Perturbation plots as a graphical representation of relative factor effects. Factors vary on the $\mathrm{X}$ axis from lowest to highest coded values. On the $\mathrm{Y}$ axis are different aspects of model response to loading: median von Mises stress and median tensile and compressive strain. This type of plot illustrates the effects of varying factors independently, i.e. one factor is varied, while other factors are kept constant at their baseline level. Horizontal lines indicate that a factor had little influence on the response, whereas a steep line indicates a strong influence. Since the responses were transformed (see text), even the responses for factors that have no interactions or quadratic components may not be straight lines. Median von Mises stresses were not sensitive to material type, and therefore only one plot is shown (with values the average of both material types). For strains, use of intensity-based materials reduced the levels of both modes of strain. Off-axis loading, independent of the direction, increased stress, with the lines not overlapping, evidencing asymmetry in vertebra shape (see Figure 4 for model cross sections under various loadings). Offset in any direction reduced stresses. Lateral and dorsal-ventral loadings had quadratic effects, and their lines do not overlap, evidencing an asymmetry in the vertebra shape. Overall process size and offset had the smallest effects, whereas loading had the largest. Most influential on the strains was the neck size, although interestingly its effects were different in tensile than in compressive strains. However, it is important to remember that the lines representing the effects of offset and dorsalventral loading can be misleading because the factors interact (Figure 5). To simplify comparison we report strain absolute values. Recall, however, that perturbation plots have to be interpreted carefully when interactions are present. 

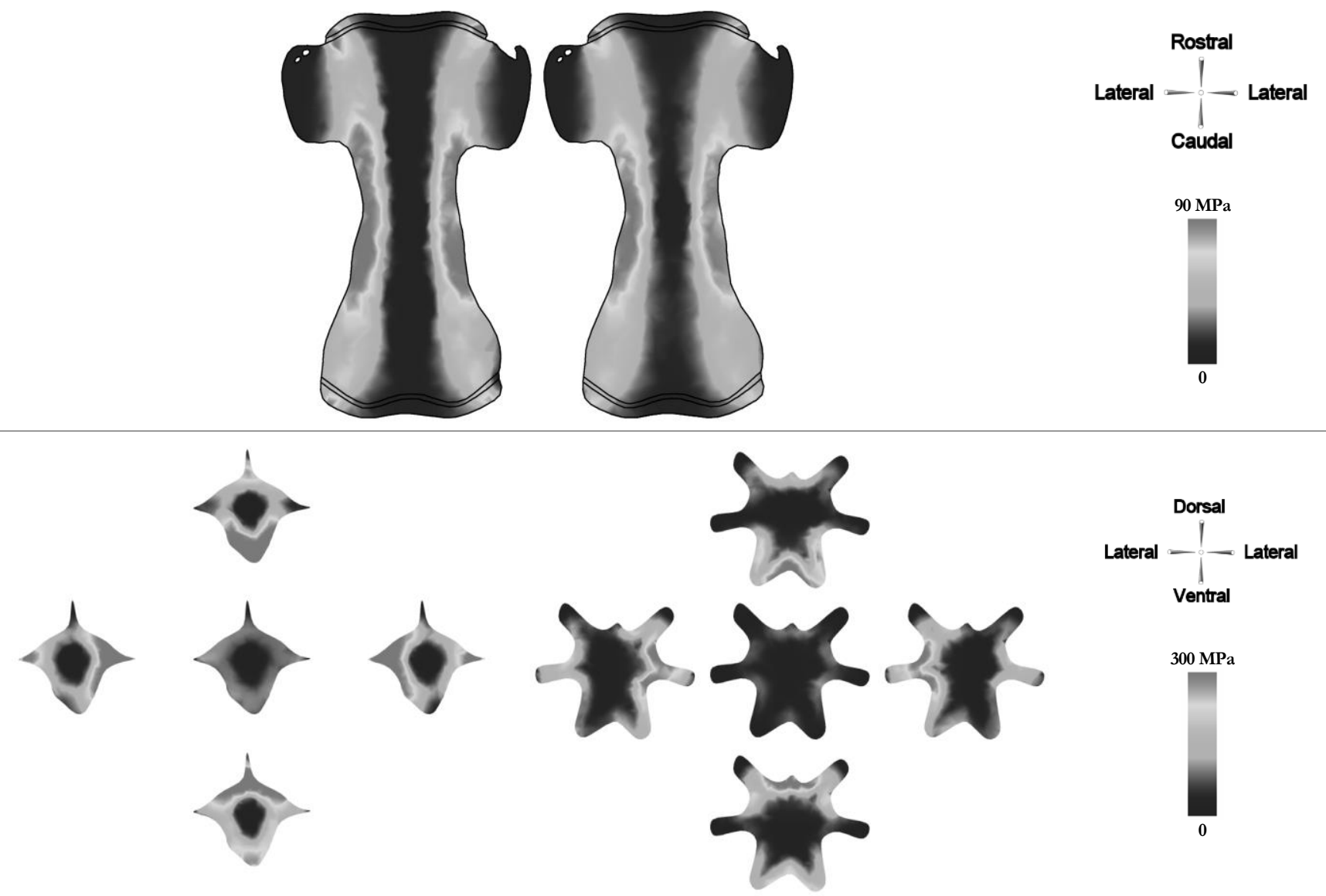

Figure 4: Effects of the material type and loading direction on the von Mises stress. Longitudinal (top) and transverse (bottom) cross sections of the baseline model colored according to the magnitude of von Mises stress. Two models with the baseline geometry and axial loading, with intensity-based (top left) and homogenized (top right) materials. Although the distributions are very similar, the model with homogenized materials presents slightly larger stresses, and somewhat larger stress gradients (see, for example, the edge of the deep blue central region, the edges are sharper on the homogenized case). Also noticeable is that the processes are under small loads in both models. Note the outlines of the growth and end plates. The effects of loading direction are illustrated with cross sections through the baseline geometry, at the level of the neck (bottom left), or through the caudal processes (bottom right). At the center is the case with axial loading, around are off-axis $( \pm 1)$ loadings laterally and dorsal-ventral. Stresses were clearly minimal under perfectly axial loading. Under off-axis loadings the deformations are a combination of compression and bending. However, both of these modes contribute to the von Mises stress. Off-axis loading increased the load carried by the processes, but the stresses were still concentrated on the base of the process, and depended little on the process shape. 
Median von Mises stress (MPa)

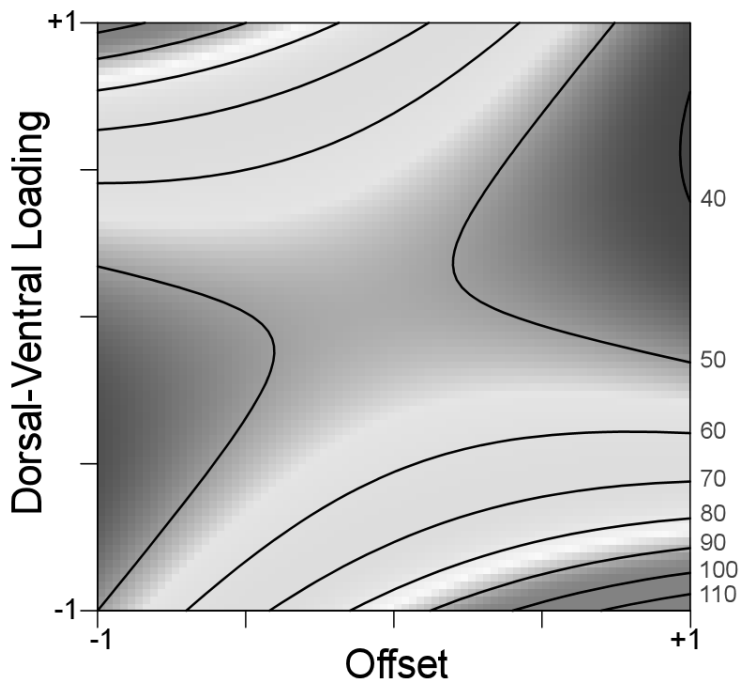

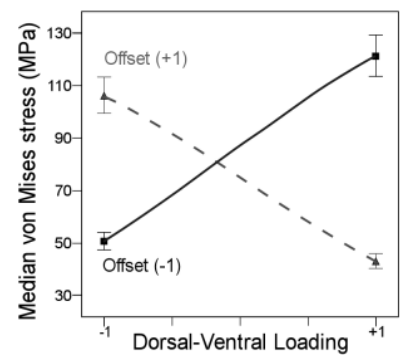

Peak von Mises stress (MPa)
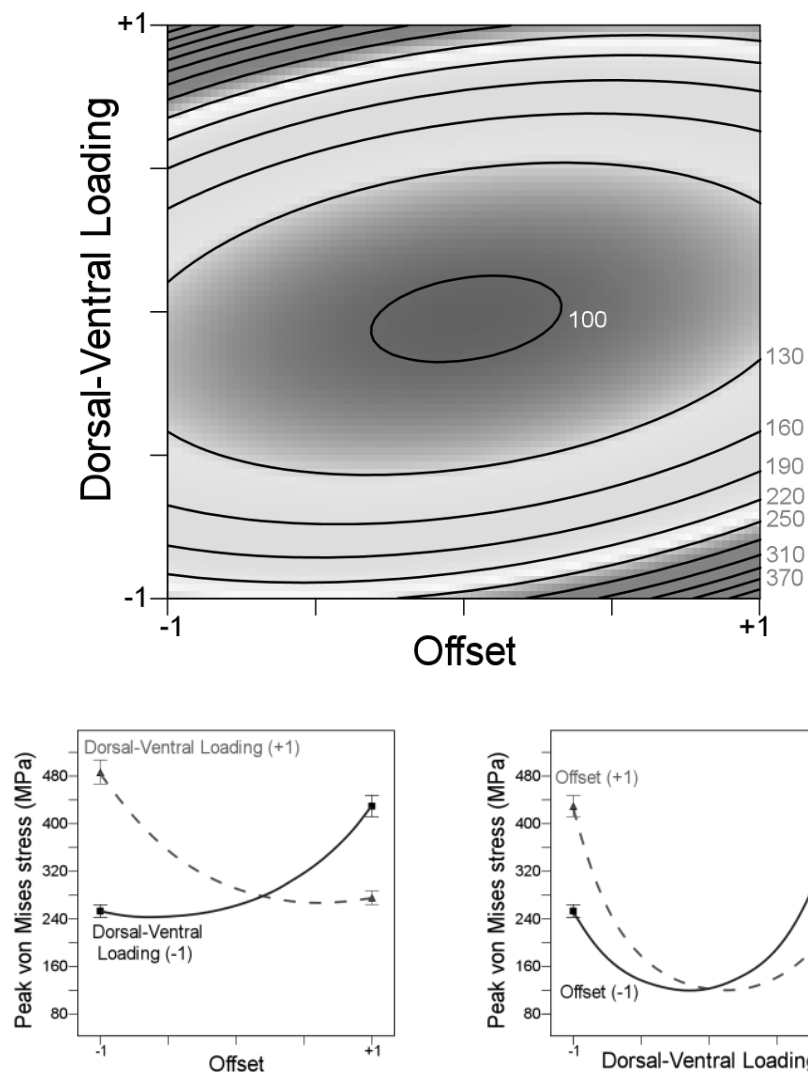

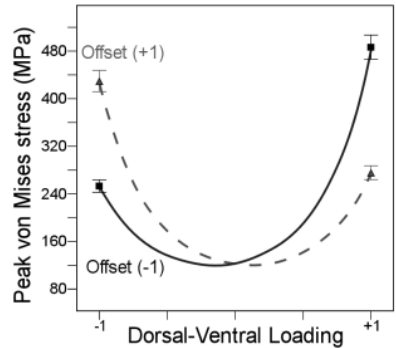

Figure 5: Effects of the interaction between offset and dorsal-ventral loading. There was a strong interaction between dorsalventral loading and offset. There are two traditional ways to illustrate the effects of an interaction like this, contour level plots (top) and so-called interaction plots (bottom). Median (left) and peak (right) stresses were affected differently by the interaction. Peak stress was minimum near the center of the factor space (the baseline). Contour levels of stress formed ellipses centered on this minimum. The ellipses were slightly tilted, explaining the asymmetry in effects of lateral and dorsal-ventral loading seen in the perturbation plots of Figure 3. The response surface for the median stresses had a saddle shape, with the inflection point at the center. This evidences a maximum in one direction, and a minimum in another. These directions were not perfectly aligned with the offset and dorsal-ventral loading. Interactions between factors are also often illustrated with interaction plots where non-parallel lines evidence an interaction and are relatively easy to distinguish. Cross lines, such as in these examples, are even clearer. For instance, it is easy to see that the effects of off-axis loading could be substantially different depending on the vertebra offset. Switching loading from one direction to the other leads to increases in stress for offset in one direction, and decreases for offset in the opposite direction. Similarly, the effects of variations in offset may depend strongly on the direction of loading. However, some characteristics of the interaction are more difficult to see in the interaction plots than on contour level plots. For example, from only the interaction plot lines it is difficult to form a mental image of the ellipses in the peak stresses, or the saddle shape on the median stresses. It is therefore common to evaluate both types of plot and pick the one that illustrates most clearly the important effects for the case at hand. 